Revue d'histoire de l'Amérique française

REVUE D.HISTOIRE DE L'AMÉRIQUE FRANÇAISE

\title{
Archives publiques du Canada. Division des archives fédérales. Documents historiques du gouvernement/Historical Records of the Government of Canada. Ottawa. 1978.61 p.
}

\section{Marcel Caya}

Volume 34, numéro 2, septembre 1980

URI : https://id.erudit.org/iderudit/303861ar

DOI : https://doi.org/10.7202/303861ar

Aller au sommaire du numéro

Éditeur(s)

Institut d'histoire de l'Amérique française

\section{ISSN}

0035-2357 (imprimé)

1492-1383 (numérique)

Découvrir la revue

Citer ce compte rendu

Caya, M. (1980). Compte rendu de [Archives publiques du Canada. Division des archives fédérales. Documents historiques du gouvernement/Historical Records of the Government of Canada. Ottawa. 1978. 61 p.] Revue d'histoire de l'Amérique française, 34(2), 275-276. https://doi.org/10.7202/303861ar d'utilisation que vous pouvez consulter en ligne.

https://apropos.erudit.org/fr/usagers/politique-dutilisation/ 


\section{COMPTES RENDUS}

Archives publiques du Canada. Division des archives fédérales. Documents historiques du gouvernement/Historical Records of the Government of Canada. Ottawa. 1978. 61p.

Depuis quelques années, la division des archives fédérales des Archives publiques du Canada a publié les inventaires généraux d'un certain nombre de fonds d'archives fédérales. En vertu de ce programme de difiusion, les inventaires généraux de ministères ou agences comme Statistique-Canada, les Affaires indiennes, le ministère du Commerce, la Gendarmerie Royale, etc. sont déjà disponibles. Afin d'aider à mieux situer ces inventaires plus détaillés dans l'ensemble de la collection, Documents historiques $d u$ gouvernement $d u$ Canada constitue un guide général qui ne vise qu'à décrire sommairement les fonds conservés par la division des archives fédérales. Une brève introduction générale portant sur l'histoire, les services et les pratiques de la division est suivie d'une liste des groupes d'archives ordonnée selon certaines catégories générales et des titres des diverses séries composant chacun des groupes d'archives. Une énumération des groupes d'archives par ordre alphabétique aide à identifier les numéros des différents groupes et facilite le repérage.

Tout en contribuant à mieux faire connaître la diversité des fonds d'archives gouvernementales gardées aux Archives publiques du Canada, ce guide constituera surtout un outil de référence précieux aux chercheurs qui possèdent déjà une bonne connaissance de ces fonds d'archives. En attendant la publication d'un plus grand nombre d'inventaires généraux, la simple énumération des titres de séries ne suffit malheureusement pas à rendre compte de la portée des divers groupes d'archives qu'il contient et servira surtout à l'initié. Une description générale du mandat et un bref historique de chacun des ministères et agences visés par les groupes d'archives auraient pu permettre de mieux évaluer le contenu et l'intérêt d'un groupe ou d'une série.

Puisque la plupart des documents d'archives fédérales sont en langue anglaise, on doit aussi se demander à quelle réalité correspond la traduction française qui est publiée conjointement avec le texte anglais original. Cet effort, rendu nécessaire par les politiques de bilinguisme du gouvernement fédéral, résulte au mieux en un produit artificiel qui oblige constamment à référer à l'original anglais pour obtenir le vrai titre de chacune des séries. De plus, des déficiences sérieuses dans la traduction rendent hasardeuse l'utilisation exclusive de cette version française. Le terme records, par exemple, est traduit indifféremment par documents, dossiers, archives. Sous la rubrique Conseil du Trésor (RG 55), la série 
National Defence Establishment n'a rien à voir avec la «Création de la Défense nationale» comme l'indique le texte, mais concerne plutôt les effectifs de ce ministère; de même sous la rubrique Vérificateur Général, on devra comprendre qu'il s'agit des Copies de lettres du Contrôleur de la Free Bank et non des documents du Contrôleur des copies de lettres de la Free Bank. Les erreurs du genre sont inévitables puisqu'il est très difficile de traduire des mots sans en connaître le contenu; il faut plutôt blâmer une application ridicule des règles du bilinguisme du gouvernement pour cet effort inutile et probablement coûteux. Le chercheur qui prévoit utiliser le guide comme outil de référence devra de toute façon s'en remettre à la version anglaise.

Tout en appréciant le travail des archivistes qui ont compilé la version originale anglaise, il convient d'insister sur le fait que, tel quel, le guide fournit encore trop peu de données utilisables pour le non-initié et qu'il faudra, pour le rendre vraiment pertinent, continuer l'effort de publication d'inventaires généraux pour au moins chacun des principaux groupes d'archives. Ce n'est qu'à ce prix que la richesse et la portée des archives gouvernementales du gouvernement fédéral peuvent être mieux conniucs ct cxploitćcs. 